\title{
Low-cost assessment tools for the development of psychomotor competency in procedural surgicall skills
}

\author{
A. Doud ${ }^{1}$, R. Dockter ${ }^{2}$, D. Vetter ${ }^{2}$, J. Rana ${ }^{2}$, M. Koester ${ }^{2}$, Z. Rzeszutek ${ }^{2}$, and J. Hjaltason ${ }^{2}$ \\ ${ }^{1}$ University of Minnesota Dept. of Biomedical Engineering, Minneapolis, Minnesota, USA \\ ${ }^{2}$ University of Minnesota Dept. of Mechanical Engineering, Minneapolis, Minnesota, USA
}

Correspondence to: A. Doud (alexdoud@umn.edu)

Received: 12 April 2013 - Revised: 26 August 2013 - Accepted: 23 September 2013 - Published: 3 February 2014

\begin{abstract}
While didactic training is a crucial element of education in the health care profession, current technologies leveraging low cost data acquisition and processing may provide an attractive alternative means for rapid, objective assessment of the foundational skills. When these technologies are leveraged towards improving the procedural surgical skill set, there is a strong opportunity for enhancing current training practices. While the proctor will still play a crucial role in the refinement of clinical judgment, affordable options for rapid training of procedural skills may serve as an avenue to free an expert proctor to focus on the more nuanced elements of medical training. Here we propose and implement a system for the low cost assessment and training of procedural surgical skills. A knot-tying module and a tool articulation module were created using affordable sensing technologies, and initial validation with a trained clinician showed that the devices could discriminate correctly and poorly tied knots. The system lays the framework for a modular experiential component of skills development in the training of health professionals.
\end{abstract}

\section{Background}

The medical training of today draws heavily from a didactic tradition of instruction, centered on a mentor-apprentice structure. While this model offers invaluable experience in real-world patient care, recent developments in medical simulation technology offer options for efficient, economical and standardized medical instruction. The push to move toward a truly evidence based medical system will depend on the ability to provide students with a set of core competencies that may be objectively assessed and improved upon. In an effort to move toward this goal, virtual reality platforms and physical training models are undergoing development and indepth investigation (Loukas et al., 2012; Morris et al., 2012; O'Connor and McGraw, 1997).

The Fundamentals of Robotic Surgery Committee (FRS), a panel of opinion leaders in robotic minimally invasive surgery, has determined that a physical trainer is most desirable for the high-stakes assessment of surgical competency (Satava et al., 2012). Physical trainers can be produced at lower cost than virtual reality platforms. Physical systems also inherently provide haptic feedback, a crucial component of clinical realism that can be difficult to produce at high fidelity in a virtual platform (Våpenstad et al., 2013). If highstakes certification will be awarded based on performance on mechanical simulators, it will be essential to provide reliable, low-cost training materials that provide students with a quantifiable means of practicing the key psychomotor competencies that will be essential for success in their profession.

A device for the assessment of surgical knot tying and a second device for measuring the accuracy of articulation of surgical tools were developed to meet the requirements put forth by the FRS committee in its most recent communication of intent for resident certification. While these modular tools can acquire the core quantitative metrics identified by the FRS committee as indicators or psychomotor surgical skill, our team has identified a wide variety of markets beyond the scope of robotic surgery, where these modules could play a key role in education for the health care professions. By making these modular components of experiential curriculum available to medical educators, we believe 


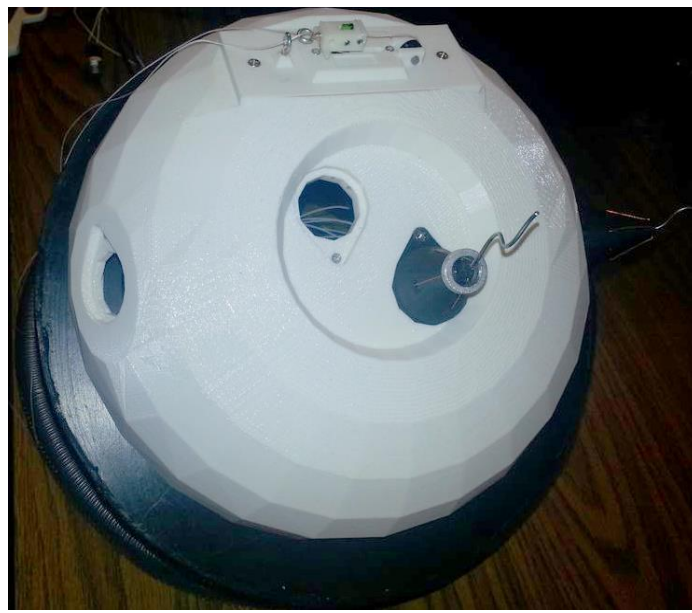

Figure 1. The integrated dome design is shown with integrated knot tying (top) and ring tower transfer (centerright) modules.

that procedural knowledge can be delivered in a standardized manner conducive to evidence-based medical practice.

\section{Design description}

Our team implemented two independent modules that were combined into a unified demonstration for a recent FRS committee meeting. For the purposes of demonstration, the modules were integrated into a dome measuring $24 \mathrm{~cm}$ in base diameter shown in Fig. 1. The dome layout was a conception of the FRS committee intended for the high-stakes certification exam, where all the modules would be operated on in sequence by the test taker and the curved surface would present an obstacle around which the test taker would maneuver. The modules we created employed mounting hardware for ease of integration or detachment from the dome demonstration. Each module sends data to an Arduino Microcontroller, enabling wired or wireless communication with a host computer. In this way, the student or faculty can easily upload the performance data for each task to a personal computer for future assessment or longitudinal evaluation. Electronic components for each module were integrated with the Arduino system using shields that can be easily added or removed from the Arduino architecture. This approach ensured a level of modular design as the system was developed. Mechanical components were developed iteratively in CAD and then printed with a 3-D printer.

A custom software solution for Arduino and PC was implemented in C++ using the cross-platform Qt library (http: //qt.digia.com, digia Helsinki, Finland). This software allows the user to manage task execution and view performance data immediately after completing the task. The software outputs text files with all relevant user metrics in a format widely accepted by available data management packages like Excel and MATLAB. The importance of data logging was stressed

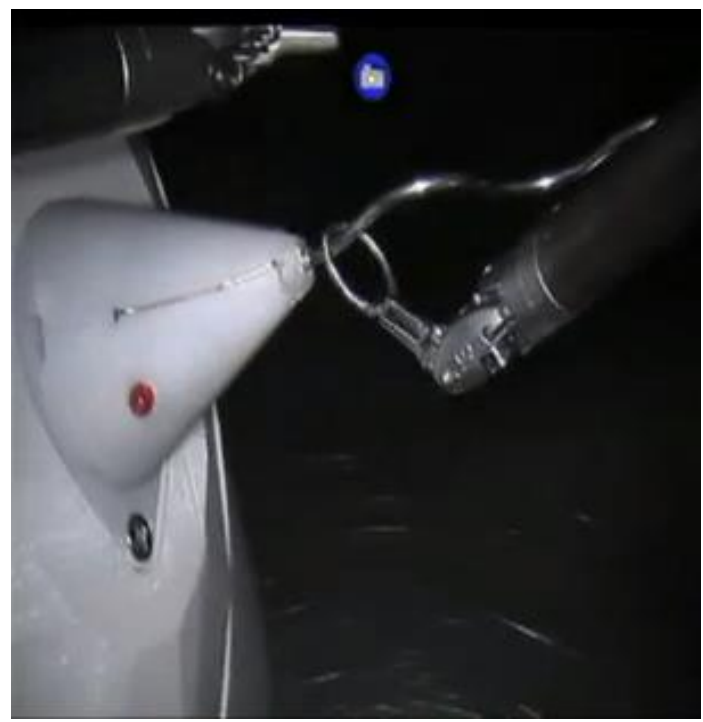

Figure 2. Ring tower transfer task as performed by a surgeon using a da Vinci robot.

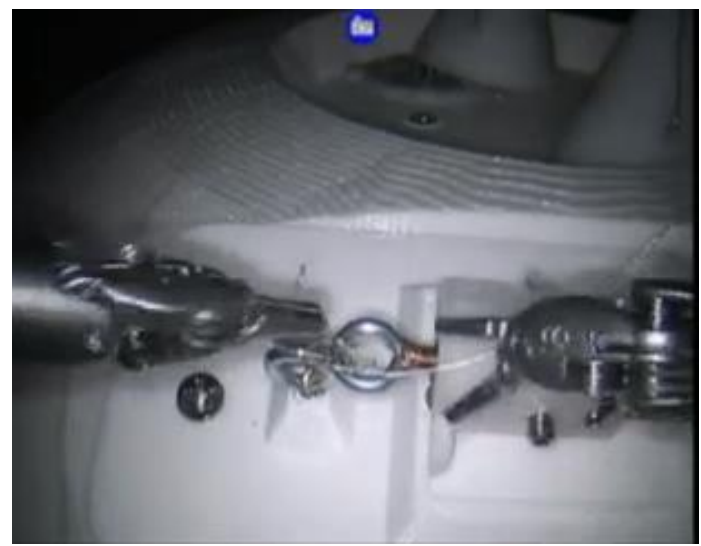

Figure 3. Proof of concept validation of the knot-tying task with a physician-controlled da Vinci surgical robot.

in our design to ensure successful data acquisition during upcoming validation studies.

\section{Tower transfer task}

The module for the assessment of accurate surgical tool articulation is shown in Fig. 2 and was realized as a set of four small S-shaped towers resting on conical bases. The student is required to remove a ring that has been threaded over the tower without coming into contact with the tower itself; the student must then pass the ring to the second tower and thread the ring over that tower without contacting it. This action is then repeated with the second two towers. The speed with which the student can complete the task was assessed via continuity checks for the ring leaving and returning to the 
conical base of the tower, and erroneous contacts with the towers were recorded via a capacitive touch sensor.

The capacity of the system to reliably detect ring contact was assessed with rings of different sizes (conductive stainless steel jump rings measuring 4, 6, 8 and $10 \mathrm{~mm}$ in diameter). Contact with robotic and laparoscopic tools in different configurations was also assessed (da Vinci grasper model 400121 and Covidien $31 \mathrm{~cm}$ Endo Grasp (TM) $5 \mathrm{~mm}$, model number 173030). The system was tuned, calibrated and debounced programmatically until no further errors were found in the ability of the device to detect erroneous contact from these sources after multiple intentional contacts of varying temporal profiles and characters. The ability of the device to quantify the time to task completion was validated by the design team members via independently measured stopwatch values.

\section{Knot-Tying task}

The module for the assessment of surgical knot tying is shown in Fig. 3 and was developed in parallel with the ring tower transfer task. Two small rings serve as the attachment points for the student's knot. When the knot is tied, the device senses the relative position of the rings and how closely they are approximated via a linear potentiometer calibrated to the task's initial position. After the knot is tied, the assessment is initiated by mechanically loading the knot with a predetermined force applied via a tunable actuator of high inter-trial accuracy. Knot security is the final position of the potentiometer divided by the initial position prior to loading, and expressed as a percent of total ring approximation (100\% when rings are touching).

Initial proof of concept testing was performed in conjunction with a practicing surgeon representing the FRS consortium. In a demonstration performed in the presence of FRS opinion leaders, the knot integrity values recorded for two correctly tied knots were 96.5 and $97.1 \%$ ring approximation. In contrast, two intentionally sub-optimal knots tied by the same physician resulted in measured post-loading approximation values of 72.9 and $74.6 \%$.

\section{Conclusions}

The presented modular solutions were designed and implemented as part of the New Product Design and Business Development course at the University of Minnesota. The critical initial design objectives set forth by the FRS committee for desired capabilities of these modular subtasks were met by the design team's implemented solutions. Since then, a broader development effort has been initiated to determine the applicability of these devices to undergraduate medical education and to training in other health service professions. Initial discussions with medical educators have identified an exciting potential for integration of the modules into medical school curricula as part of a content delivery system for procedural medical knowledge.

Acknowledgements. The team would like to thank our collaborators at the University of Minnesota. We extend a special thanks to Tim Kowalewski, the MRD lab, Robert Sweet and the CREST Center.

Edited by: D. Brouwer

Reviewd by: two anonymous referees

\section{References}

Loukas, C., Nikiteas, N., Schizas, D., Lahanas, V., and Georgiou, E.: A head-to-head comparison between virtual reality and physical reality simulation training for basic skills acquisition, Surg. Endosc., 26, 2550-2558, 2012.

Morris, M., Gallagher, T., and Ridgway, P.: Tools used to assess medical students competence in procedural skills at the end of a primary medical degree: a systematic review, Med. Educ. Online, 17, 18398, doi:10.3402/meo.v17i0.18398, 2012

O'Connor, H. M. and McGraw, R. C.: Clinical skills training*: developing objective assessment instruments, Med. Educ., 31, 359363, 1997.

Satava, R. Smith, R., and Patal, V.: Fundamentals of Robotic Surgery: Outcomes Measures and Curriculum development, White Paper: FRS Summary, Distributed at SLS 2012, Boston MA, 2012.

Våpenstad, C., Hofstad, E. F., Langø, T., Mårvik, R., and Chmarra, M. K.: Perceiving haptic feedback in virtual reality simulators, Surgical Endoscopy, 27, 2391-2397, 2013. 